Fecha de recepción: marzo 2021 Fecha de aceptación: abril 2021 Versión final: mayo 2021

\section{El Taller de Diseño Centrado en el Usuario}

Ivonne Ortiz-Sánchez M.A. Phd (c) ${ }^{(1)}$

\begin{abstract}
Resumen: Este artículo se enfocará en la asignatura de Taller de Diseño Centrado en el usuario (TDCU), para analizar las propuestas teóricas, pedagógicas y metodológicas que se han construido dentro de la asignatura, así como sobre el proyecto de Diseño con énfasis en el usuario. Las estrategias metodológicas y pedagógicas aplicadas para la enseñanza del Diseño y los resultados obtenidos de este proceso de construcción de conocimiento, así como los aciertos y errores que se han generado durante la implementación de esta asignatura, en los cuatro periodos que ha sido impartida de manera presencial y virtual. Se realiza un recuento sobre la Carrera de Diseño de Productos y la construcción del Rediseño de la cual nace la propuesta del TDCU, a partir de esto se conoce la estructura de la asignatura; se desarrolla los principios pedagógicos basados en el cognoscitivismo que se utilizan en esta cátedra, además de la propuesta metodológica que se aplica en este proceso de enseñanza y adquisición de conocimiento. Una vez desarrollado este marco se trata sobre las estrategias adoptadas para la virtualización de la asignatura, los retos enfrentados, los aciertos alcanzados, las limitaciones y errores cometidos, para analizar y concluir el futuro de la asignatura.
\end{abstract}

Palabras clave: Taller de Diseño Centrado en el Usuario (TDCU) - Metodología - Resultados de aprendizaje - Pedagogía.

[Resúmenes en inglés y portugués en las páginas 138-139]

(1) Ivonne Ortiz-Sánchez M.A. Phd (c), Pontificia Universidad Católica del Ecuador.

Adscrita a la Pontificia Universidad Católica del Ecuador, en la Carrera de Diseño de Productos de la Facultad de Arquitectura, Diseño y Artes. Graduada en Diseño con mención en Productos por la Pontificia Universidad Católica del Ecuador (PUCE). Estudió en la Universidade de Lisboa en la Faculdade de Belas Artes (FBAUL), obteniendo el título de Mestre em Design de Equipamento com especialização em Estudos de Design. Actualmente se encuentra como Phd candidate en el XXXV Ciclo del Doctorado Internacional de Arquitectura y Planificación Urbana de la Universidad de Ferrara (UNIFE). Es docente a tiempo completo de la Carrera de Diseño de Productos de la FADA-PUCE desde el 2015 y docente a tiempo parcial de la FAU-UCE desde el 2014. Investigadora sobre temas relacionados a la sostenibilidad en él diseño, ética, filosofía, teoría, metodologías y crítica del diseño. Código ORCID: 0000-0002-5138-1486 


\section{Introducción}

Este artículo se enfocará en la asignatura de Taller de Diseño Centrado en el Usuario (TDCU), para analizar las propuestas teóricas y metodológicas que se han construido dentro de la asignatura, así como sobre el proyecto de Diseño con énfasis en el usuario. Las estrategias pedagógicas y metodológicas aplicadas para la enseñanza del Diseño y los resultados obtenidos de este proceso de construcción de conocimiento, así como los aciertos, limitaciones y errores que se han generado durante la implementación de esta asignatura en los cuatro periodos que esta ha sido impartida de manera presencial y virtual.

\section{La Carrera de Diseño de Productos de la FADA-PUCE}

Para comprender de donde nace este Taller y su propuesta, es necesario resaltar que la Carrera de Diseño de Productos ha tenido diferentes perspectivas que se han aplicado de acuerdo con el contexto temporal y de desarrollo de la profesión en Quito-Ecuador. La Carrera como tal cuenta con 27 años de experiencia y nació con una visión global tomando el nombre de Diseño Integral, en donde los estudiantes se formaban con un conocimiento global en varios ámbitos del Diseño, especialmente en el área de Diseño de Productos y Gráfico. Con el pasar de los años, la carrera se especializó pasando a tener énfasis solo en el desarrollo de productos, y distinguiéndose de Diseño gráfico (Licenciatura en Diseño con mención en Diseño de Productos). El enfoque de los talleres de la carrera se centró, por ende, en el desarrollo de proyectos de diferente índole como mobiliario, luminarias, indumentaria, accesorios, transporte, menaje de cocina, entre otros; esta formación se enfocaba en los tipos de objetos, su escala y complejidad; basada en la propuesta de Sánchez (2005):

Con referencia a los objetos de escala háptica "[...] comprende la gestualidad como manera de comunicación (con énfasis en la escala de las manos), y la forma en cuanto a mediadora de actos y gestos es una estructura háptica. De este estudio deberían proceder los objetos de la tecnología digital y no de la "espichadera de botones" (un cubo de Rubick, una taza o una joya son estructuras hápticas)"; proxémica "comprende las relaciones entre los individuos y el espacio o de cómo los individuos comprenden el espacio (entrar, salir, pasar, desplazarse, adentro, afuera, fluir, etc.) y la forma como espacio estable o configuración de un espacio es una estructura proxémica. Es muy utilizado en los puestos de trabajo, cabinas y todos los objetos de inclusión, macroobjetos y objetos semimóviles que tienden a contener o semicontener el cuerpo humano y que son de orden espacial" (2005, p. 31); ...cinético "comprende todo lo relacionado con el movimiento, en donde la forma o condiciona movimiento desde su morfología (perceptivo, tensiones formales) o funciona como medio de generación de movimiento (una patineta)”. (2005, p.32) 
Sucesivamente, durante la construcción y desarrollo del Rediseño de la Carrera de Diseño de Productos entre el 2015 y 2018, los talleres son organizados con el objetivo de la construcción de conocimiento acumulativo, además de los énfasis necesarios en el contexto actual: estética, usuario, materiales y procesos productivos, diseño social (para y con las personas), diseño de servicios (productos intangibles) y diseño estratégico (empresa). Esta propuesta se basa en el hecho de que el proyecto de diseño puede desarrollarse desde varias visiones y que cada una de estas tiene su proceso metodológico, además que sus propias herramientas y métodos, o énfasis para la aplicación de estas herramientas; con la finalidad de que el estudiante desarrolle la capacidad de discernir sobre los proyectos que enfrentará y como los abordará.

Posteriormente a la aprobación del mencionado rediseño en el 2018, se planteó una visión vertical a través de los Talleres. El objetivo de esta propuesta fue el de que el conocimiento fuese acumulativo, y buscase aplicarse, combinarse y sustentarse con lo aprendido en módulos o talleres en los semestres precedentes; además de manera transversal, a través de ejercicios que recibiesen el aporte de una o varias asignaturas y de un proyecto integrador. Ya que el Taller de Diseño Centrado en el Usuario (TDCU) es impartido en el tercer nivel, es necesario especificar que a este le preceden los talleres o módulos de Fundamentos del Diseño y de Diseño Centrado en el Producto, a los que se ha hecho referencia previamente y que constituyen los conocimientos base de partida.

En el taller de Fundamentos del diseño se adquieren herramientas teórico-prácticas para entender y aplicar los fundamentos básicos del diseño: forma, espacio, color y signo. El propósito es comprender el proceso de concepción y tangibilización de objetos bidimensionales y tridimensionales, mediante procesos sistémicos y principios fundamentales de diseño, que respondan a problemas o necesidades reales en torno a un objeto. El Taller de Diseño Centrado en el Producto, por otro lado, tiene un enfoque estético y se orienta a la introducción y manejo de herramientas para el desarrollo de productos de uso cotidiano vinculado a un análisis técnico y tecnológico de sus componentes y aplicaciones. Durante estos dos módulos se desarrollan proyectos de mediana complejidad y valor innovativo, orientados al uso, forma, función y expresión del objeto dentro de un entorno cultural, económico y tecnológico local. (PUCE, 2018, pp. 3-4)

\section{Estructura del Taller de Diseño Centrado en el Usuario}

El TDCU se enfoca en la comprensión de las necesidades y comportamiento del ser humano. Se analizan las decisiones, deseos y limitaciones de los usuarios como criterios fundamentales para la configuración de un producto. Se profundiza en principios, teorías e instrumentos de Diseño Centrado en el Usuario y Diseño Emocional que faciliten la toma de decisiones en un proyecto de diseño. (PUCE, 2018, p. 5). Además de adquirir nuevos conocimientos, los estudiantes suman al proyecto, el conocimiento de los talleres anteriores: específicamente los principios de configuración y el dominio de la estética.

Dentro de los resultados que se buscan alcanzar están los siguientes: (1) usar las bases teóricas y conceptuales del Diseño para el usuario, en propuestas de diseño de productos 
funcionales; (2) desarrollar la capacidad de conceptualizar, investigar y argumentar proyectos en torno al usuario; (3) Aplicar criterios de identidad y expresión a los productos (criterios estéticos); (4) aplicar datos y variables ergonómicas en el diseño de productos (ingeniería de factor humano, análisis perceptual, diseño de productos culturales, la psicología cognitiva, y el diseño de usabilidad); (5) realizar un trabajo interdisciplinario para la configuración y validación de productos mediante el Modelo de Diseño Centrado en el Usuario y el Diseño Emocional; (6) interpretar la sensibilidad de los objetos y su relación con los usuarios y sus particularidades.

La estructura del TDCU se articula en cuatro partes: la primera trata sobre los conceptos básicos como la definición y las características básicas, usabilidad, principios de diseño universal, diseño emocional y experiencia del usuario (UX). La segunda parte se enfoca en la investigación en el DCU para conocer los contextos, hábitos y conductas de usuarios, los métodos y herramientas de investigación del DCU, definición y tipos de usuario y requerimientos del proyecto. La tercera parte busca el desarrollo creativo en el DCU, mediante el cual se aborda el concepto de diseño y los métodos creativos. Finalmente, la cuarta parte está centrada en la comprobación en el DCU y en los métodos de evaluación de diseño universal, diseño emocional, usabilidad y experiencia de usuario. (PUCE, 2019, pp. 1-8)

\section{Fundamentos teóricos}

Dentro del TDCU utilizamos como referencia los textos Diseño interactivo, teoría y aplicación del DCU de Pratt, A. y Nunes, J. (2013) para los conceptos y características; y Beautiful users de Lupton, E., Carpentier, T., y Lambert, T. (2014) para ilustrar la historia del DCU y el diseño para las personas. A continuación, ejemplificamos los conceptos base.

La definición del DCU es la descrita por Pratt, A. y Nunes, J. (2013) “El Diseño Centrado en el Usuario o DCU (del inglés UCD User-Centered Design) es un enfoque del diseño que se centra en el usuario de un producto o una aplicación para crear un determinado producto digital. El DCU implica que el diseñador estudie a fondo las necesidades, los deseos y las limitaciones del público objetivo al que va dirigido el producto final, y a partir de ese análisis toma las decisiones que procedan para confeccionar su creación." (p.12)

En cuanto a los usuarios, su propuesta es generar una pirámide de prioridad de usuarios, en la cual hay que identificar quienes y de qué manera intervienen en el proyecto. Esta pirámide se compone de: el grupo objetivo, o los usuarios tipos, que son los que intervienen directamente con el objeto y que interactuaran constantemente con él; los usuarios que no son parte del grupo objetivo que intervienen indirectamente; los usuarios que tienen experiencias extremas, completamente diferentes a las del usuario tipo y los expertos que son profesionales interdisciplinarios que aportan con información específica y técnica al proyecto. 
Para las definiciones de uso, usabilidad, experiencia, diseño emocional e interacción se utiliza: Design by use de Brandes, U., Stich, S.; Wender, M. (2013); el Handbook of usability de Rubin, J., y Chisnell, D. (2008); Living with complexity y Emotional design, why we love or hate everyday thingns ambos de Norman, D. $(2007,2016)$. A continuación, revisamos los conceptos de uso y usabilidad propuestos y utilizados en clase.

Use makes the user enter into a specific type of object relationship that is located between form and function. The familiar conditions of form and function seem to disintegrate when considering an object's use because, "in principle, we can use everything for anything in a more or less successful way. Contrary to a dedicated function, use is not the entelechy of an object. If something is used for purposes other than those it was intended for, then a purpose different from the one defined by a standardized function has been chosen. (Brandes, U., Stich, S.; Wender, M. 2013, p. 55)

Usability is a quality that many products possess, but many, many more lack [...] To be usable, a product or service should be useful, efficient, effective, satisfying, learnable, and accessible [...] Usefulness concerns the degree to which a product enables a user to achieve his or her goals and is an assessment of the user's willingness to use the product at all. Efficiency is the quickness with which the user's goal can be accomplished accurately and completely and is usually a measure of time [...] Effectiveness refers to the extent to which the product behaves in the way that users expect it to and the ease with which users can use it to do what they intend [...] Learnability is a part of effectiveness and has to do with the user's ability to operate the system to some defined level of competence after some predetermined amount and period of training (which may be no time at all) [...] Satisfaction refers to the user's perceptions, feelings, and opinions of the product [...] Accessibility and usability are siblings. In the broadest sense, accessibility is about having access to the products needed to accomplish a goal. B (Rubin, J., y Chisnell, D., 2013, p. 3-4)

Cuando hablamos sobre uso, usabilidad, experiencia, emoción e interacción, estos conceptos van ligados al desarrollo de la forma y la función como objetivo del producto, además de la praxis mediante la cual alcanzamos el objetivo propuesto. Estos conceptos se refuerzan constantemente durante todo el TDCU, durante las diferentes fases del proyecto, con la finalidad de que los estudiantes identifiquen como aplicarlos y así alcanzar comprensión y aprendizaje.

En cuanto al proceso proyectual utilizamos las IDEO Method Cards (2003) y el Delft Design Guide (2017), estas dos herramientas brindan una fácil comprensión de los métodos y su aplicación, ejemplificando con casos de estudios que posibilita su comprensión. A continuación, se muestra la estructura de un método: breve descripción u objetivo, cuando y como se utiliza, el posible proceso ya que este puede variar, las limitaciones que tiene y los tips o consejos sobre este, el ejemplo es el método de desarrollo creativo Analogía y Metáfora. 
Analogías y metáforas. Podemos usar las analogías y las metáforas para buscar inspiración.

¿Cuándo?

(1) Durante la generación de ideas. (2) Las analogías son utilizadas para la conceptualización, empezando por la clara definición de un problema. (3) Se pueden utilizar analogías cercanas o lejanas al problema. (4) Las metáforas son utilizadas para encuadrar el problema y analizar. (5) Son utilizadas para comunicar mensajes particulares a los usuarios. (6) Al utilizar metáforas la inspiración debería ser posible relacionar.

¿Cómo?

(1) Buscar material que nos inspire. (2) Si queremos más creatividad, podemos buscar en dominios lejanos. (3) Cuando se encuentre el material, cuestionarse porque se asocia este recurso de inspiración con la propuesta de diseño. (4) Luego se decide cual se va a implementar, analogía o metáfora en la nueva solución de diseño. (5) Cuando se realice analogías tener cuidado en no copiar o trasladar los atributos a la solución de diseño. (6) Buscar cambiar y potenciar las soluciones a través de la abstracción y transformación.

Posible proceso (es adaptable)

(1) Encuadre (a) Analogía: el problema de diseño a ser resuelto. (b) Metáfora: cualidades de la experiencia que se quiere brindar al usuario a través de la solución del problema de diseño.

(2) Búsqueda (a) Analogía: situaciones o ejemplos donde el problema haya sido resuelto exitosamente. (b) Metáfora: concreta de una entidad que tenga las cualidades que se quiere transmitir.

(3) Aplicación (a) Analogía: Recuperar y procesar los recursos del campo de inspiración. Abstraer, transformar y transferir a la solución del problema de diseño. (b) Metáfora: Recuperar los recursos del campo de inspiración. Abstraer la esencia de las propiedades.

(4) Transformarlas para la aplicación en la solución del problema.

Limitaciones

(1) Puede llevar mucho tiempo encontrar e identificar las fuentes adecuadas de inspiración e información. (2) Identificar si este recurso puede ser utilizado en el proyecto de diseño.

Tips

(1) Utilizar analogías cercanas y lejanas, el éxito está en la abstracción y transformación. (2) Buscar las cualidades que se quiere enfatizar en el objeto para el uso de las metáforas. Trabajar en las referencias, hacerlas sutiles y no tan obvias. (Technische Hogeschool Delft, 2017, pp. 113-114) 


\section{ANALOGIAS Y METÁFORAS}

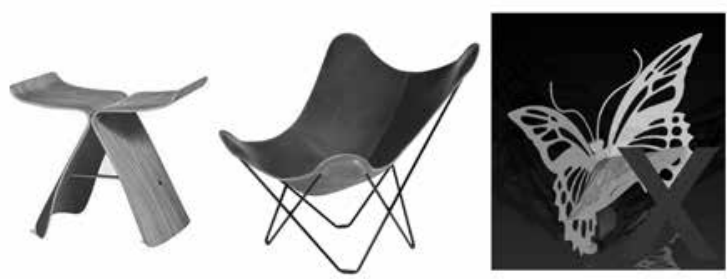

Figura 1. Ejemplo de la aplicación de métodos en mobiliario icónico de diseño. (Autor, 2020)

Los objetivos de este taller se enfocan en (1) la aplicación de los fundamentos teóricos del DCU; (2) identificar los tipos de usuarios que intervienen en los proyectos que se va a desarrollar, una vez identificados reconocer qué tipo de información se va a obtener de cada uno y cuáles son las mejores herramientas y métodos para utilizar. Busca también, (3) entender que cada método tiene un objetivo que se plantea, un proceso que se desarrolla, el análisis de la información obtenida y un resultado.

En cuanto a los estudiantes, se busca (4) incentivarles a probar y aplicar los conocimientos y hacerlo de la mejor manera posible así se falle (esto es fundamental en el proceso de aprendizaje). (5) Generar análisis de resultados que permitan reflexionar sobre la correcta aplicación del método, reconocer los aciertos y los errores e identificar la cantidad y calidad de la información obtenida, además de permitir la exploración de diferentes herramientas para ampliar la investigación que se está desarrollando.

Sobre el proceso de desarrollo creativo (6) se busca romper los esquemas preestablecidos durante la fase de investigación. Es necesario, por ende, (7) considerar los métodos creativos pertinentes para cada proyecto y (8) explorar ejercicios de creatividad para incentivar el ejercicio cerebral.

Con relación a la fase de comprobación, el objetivo es (9) aprender que cada etapa del proyecto necesita una validación y que es constante, cada paso permite entender si se avanza en la dirección correcta o identificar los cambios pertinentes. Es necesario (10) asumir que el proceso de diseño es iterativo y busca (11) desarrollar modelos y prototipos rápidos para las validaciones, además de (12) aprender que el proceso de detalle y especificación se realiza profundizando los cambios y ajustes necesarios. Es menester tomar en cuenta que, (13) dado el énfasis del TDCU, las comprobaciones de usabilidad, experiencia y emoción son indispensables. (14) Es necesario considerar que el proceso iterativo nos permite identificar oportunidades y aprendizaje en los errores cometidos para generar un resultado sólido a través de la toma de conciencia, la reflexión y la crítica. Finalmente, cabe señalar que (15) experimentar en la academia es la oportunidad para adquirir conocimiento, destrezas y capacidades que serán aplicadas en la vida profesional.

A continuación, profundizaremos en el proceso pedagógico y metodológico propuesto en el TDCU. 


\section{Proceso pedagógico}

El aprendizaje de las personas depende, no tanto de los retos e incertidumbres que contenga su mundo, sino de cómo se perciben. Incluso el entorno más cotidiano está lleno de cosas que se pueden tratar como problemáticas, si las vemos como tales. (Claxton, 2001, p. 211)

Los principios pedagógicos que se trabajan dentro de esta asignatura son los del cognoscitivismo, donde el aprendizaje es el proceso mediante el cual se crean y modifican las estructuras cognitivas. Estas estructuras constituyen el conjunto de conocimientos sistematizados y jerarquizados, almacenados en la memoria que le permiten al sujeto responder ante situaciones nuevas o similares. De ahí que, el centro principal de esta corriente es saber cómo el hombre procesa la información, que operaciones psíquicas intervienen para codificar los conocimientos, como se organizan los datos obtenidos por medio de la percepción durante los procesos de interacción con el medio y los demás seres humanos (Duran 2004, p. 34)

Para el proceso de aprendizaje es importante el conocimiento acumulado de manera consciente e inconsciente por los alumnos en su vida y en su formación profesional. Al impartirles nuevos saberes se busca motivar la asociación de estos con sus experiencias previas, hacerlas conscientes y generar un aprendizaje significativo, además de incentivar la reflexión. Es necesario, por ende, motivar el proceso cognitivo del cerebro de los estudiantes, a través del diálogo y del debate de los nuevos conceptos, para que a través de este proceso, puedan recuperar experiencias, objetos e imágenes de la memoria, como estrategia de reconocimiento del contexto. Además, esto provoca en los alumnos la búsqueda de referencias, la permanencia en un estado de atención e incentiva la observación de su entorno, haciéndolos más activos.

La corriente cognitiva estudia procesos mentales superiores en los que el pensamiento, los recuerdos y la forma en que se procesa la información repercuten en las respuestas que va a realizar el sujeto para la solución de una situación o problema. Además, considera que el sujeto aprende activamente por cuanto participa en las experiencias, busca información, y reorganiza lo que ya conoce para aumentar su comprensión

El aprendizaje como proceso mental activo de adquisición, recuerdo y utilización de conocimientos implica la participación de las sensaciones, la percepción, la atención, la memoria y del pensamiento. La mente humana, igual que una computadora, una vez que recibe información, realiza operaciones (análisis, interpretación) para cambiar su forma y contenido, la almacena, la recupera cuando necesita y finalmente genera respuestas. (Duran 2004, p. 34-35)

Los tipos de aprendizajes que se aplican y procuran dentro de esta asignatura son el repetitivo, el significativo, receptivo y por descubrimiento. 
Aprendizaje repetitivo. Este tipo de aprendizaje se pone de manifiesto cuando el alumno realiza asociaciones arbitrarias con respecto al nuevo conocimiento. Ocurre porque el sujeto no tiene conocimientos previos básicos que le permitan comprender lo nuevo, razón por la cual memoriza arbitraria y literalmente dando como resultado un aprendizaje mecánico [...]. (Duran 2004, p. 35)

Este tipo de aprendizaje se lo aplica dentro de la etapa de investigación, desarrollo creativo y comprobación en el TDCU, en la fase de aplicación de métodos. Ya que los estudiantes necesitan aplicar los métodos dentro del proyecto, es necesario un proceso mecánico que les permita superar el miedo a experimentar y fallar. Este proceso les permite desarrollar la confianza en si mismos para probar algo nuevo y obtener un resultado, así sea erróneo. Una vez realizados los ejercicios de manera individual y grupal se analizan los resultados, los aciertos y errores que les permiten acrecentar su formación y su aprendizaje.

Aprendizaje significativo. Este tipo de aprendizaje ocurre cuando los nuevos conocimientos se relacionan en forma clara y sustancial con lo que el alumno ya sabe, es decir cuando el nuevo aprendizaje se relaciona eficazmente con las estructuras del conocimiento que posee el sujeto que aprende.

Para que el aprendizaje significativo ocurra, es necesario la presencia de las siguientes condiciones:

(1) El contenido del aprendizaje dese ser potencialmente significativo, lo que significa que debe permitir ser asimilado de manera significativa. (2) El alumno debe poseer en sus estructuras cognitivas los conceptos básicos, previamente formados, de manera que el nuevo conocimiento pueda vincularse con el anterior en forma representativa y comprensible. (3) El alumno debe mostrar una actividad positiva hacia el aprendizaje significativo, es decir, debe estar dispuesto para relacionar el material de aprendizaje con las estructuras cognitivas que posee. (Duran 2004, p. 35-36)

El aprendizaje significativo en este Taller se aplica dentro del proceso proyectual y la configuración del producto. Los estudiantes conocen las fases del proyecto y los elementos necesarios para configurar el producto, temas que han venido trabajando tanto en Fundamentos del Diseño, así como el Taller de Diseño Centrado en el Producto. Saben cuáles son las etapas y el proceso, pero no conocen el énfasis del usuario, por ende, aplican los nuevos conceptos combinándolos con los conceptos previos, produciendo acumulación de conocimiento y nuevo conocimiento.

Aprendizaje receptivo. Se llama así porque el contenido total de lo que se va a aprender se le presenta al alumno en forma acabada. En la tarea de aprendizaje, el alumno no tiene que hacer ningún descubrimiento independiente. Se le exige solo que internalice el material que se le presenta de modo que pueda recuperarlo en fecha futura. (Conferencia, libro y video científico). (Duran 2004, p. 37) 
Este tipo de aprendizaje se aplica en la primera etapa de conceptos básicos, ya que aparte de la información proporcionada por la docente, los estudiantes exploran los conocimientos a través de los libros, artículos y videos. De esa manera pueden contrastar los diferentes conceptos emitidos por los autores y generar un criterio propio.

Aprendizaje por descubrimiento. En este tipo de aprendizaje, el contenido principal de lo que va a ser aprendido no se da, sino que tiene que ser descubierto por el alumno antes de ser asimilado. La tarea de aprendizaje consiste en descubrir algo. (Duran 2004, p. 37)

Este aprendizaje se logra por reflexión, tanto en la parte de conceptos básicos, así como en la investigación del DCU. Durante las asesorías o tutorías sobre el proyecto, la docente y el estudiante van a analizando mediante el diálogo, los hallazgos realizados como resultado de la investigación y la aplicación de los métodos. Finalmente, cabe resaltar que, de la mano con la pedagogía va el proceso metodológico: a continuación, se expondrá la metodología que se ha propuesto dentro de la asignatura para alcanzar los resultados de aprendizaje además del desarrollo proyectual.

\section{Metodología}

[...] known is used to generate something new that will provide answers to questions inspired by the felt need. (Owen 1998, p. 90)

Conocemos que el Diseño como disciplina única, tiene sus propios métodos y metodologías para desenvolver el proyecto de diseño, así mismo, para desarrollar su campo teórico debe adaptarse y configurarse para crear su propio proceso; de igual manera para el proceso de enseñanza. Nos hemos basamos en el artículo Building the Knowledge Base de Charles Owen como base metodológica para el desarrollo de la asignatura de Taller de Diseño Centrado en el Usuario.

Knowledge is generated and accumulated through action. Doing something and judging the results is the general model. [...] the process is shown as a cycle in which knowledge is used to create works, and works are evaluated to build knowledge. (Owen, 1998, p. 85) 


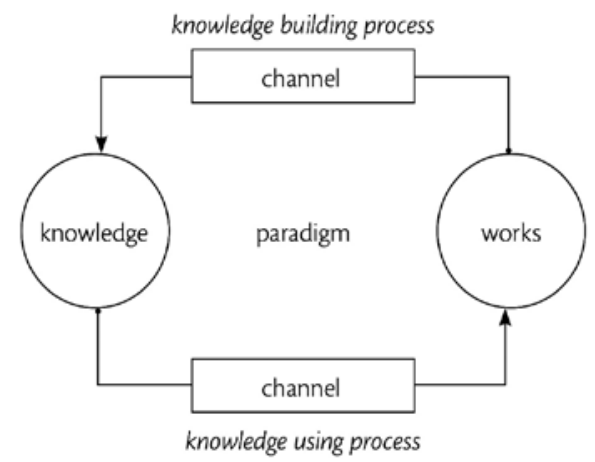

Figura 2. Modelo general para generar y acumular conocimiento. (Owen 1998, p. 86)

Este esquema (figura 2) nos muestra a modo generalizado el proceso para generar y acumular conocimiento que es: el conocimiento que tienen los estudiantes, construido en los semestres precedentes y el impartido dentro de la asignatura, este es el punto de partida para crear trabajos 'works' (práctica) que después en un proceso de evaluación crea, acrecienta o confirma el conocimiento que originó el proceso, generando una base científica y no empírica.

El esquema (figura 3) dentro del Diseño, proyecto y el proceso de enseñanza del diseño se lo aplica en dos momentos, en el ámbito analítico que es la parte de teoría "[...] the realm of theory, the model is a paradigm for inquirí. Existing knowledge, under the direction of theory, is used to generate proposals. Proposals are tested with measures that verify or refute conclusions to build knowledge". (Owen 1998, p. 86); esta es la base de conocimiento que estamos construyendo conocimiento-propuestas-verificación-mayor conocimiento; y el Sintético, ligado a la práctica y el proceso, es decir al proyecto de diseño "[...] the realm of practice, the model forms a paradigm for application. Here, knowledge is used through the application of principles to produce works. Works are judge for their worth as additions to the knowledge base using the criteria of the discipline". (Owen 1998, p. 86-87)

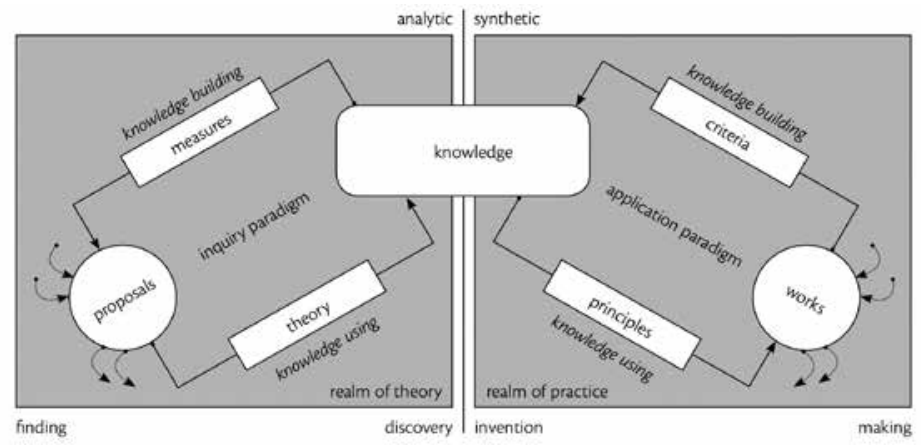

Figura 3. Usar y acumular conocimiento en los dos campos. (Owen 1998, p. 86). 
Como proceso analítico se ha propuesto que empieza con el conocimiento adquirido en los talleres previos y el impartido, la explicación de la docente, la labor del estudiante en la fase de conceptos básicos es realizar investigación para confirmar, discernir y generar un criterio: entender qué es el DCU, sus características y los conceptos que intervienen, es decir el proceso analítico. Para ilustrarlo, revisaremos el aula virtual, los recursos facilitados y los resultados obtenidos.

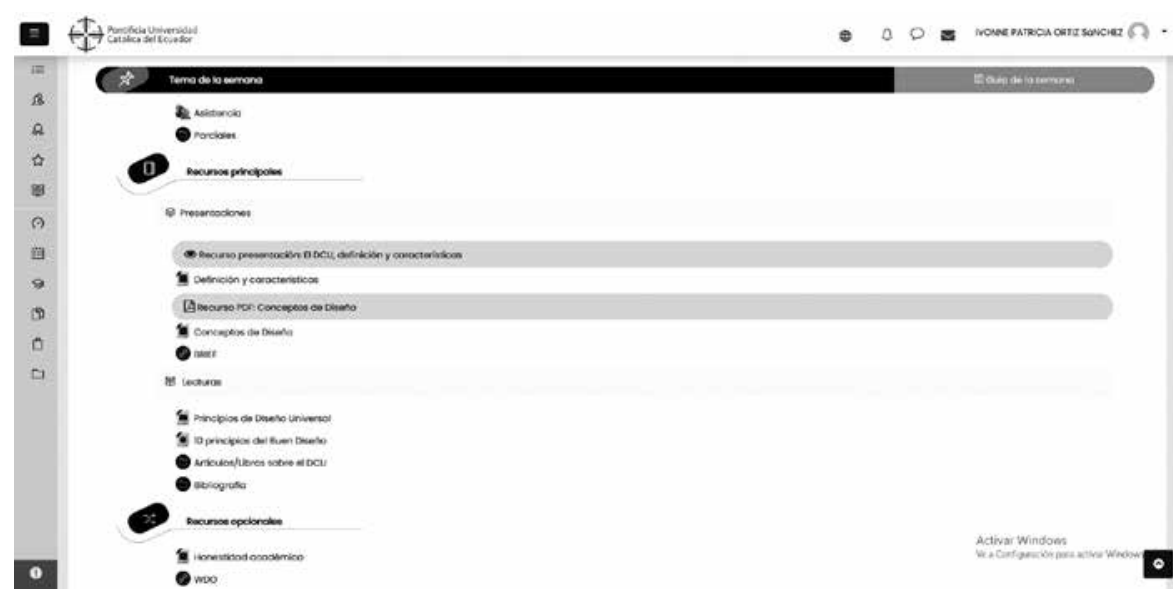

4

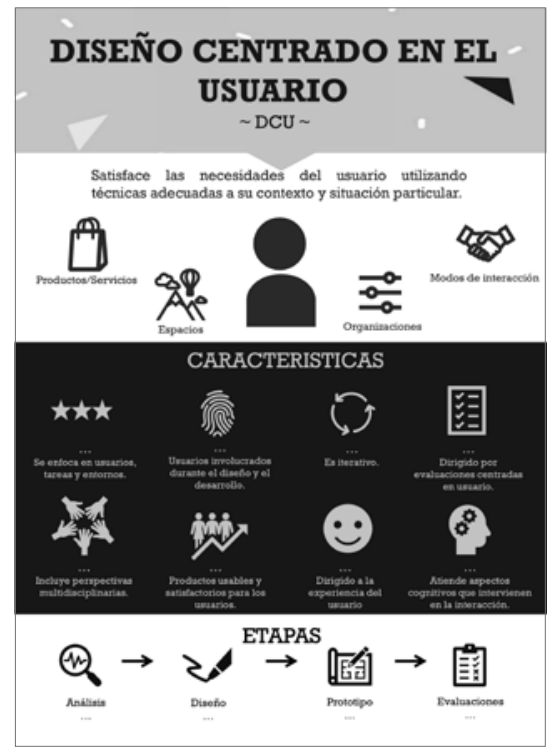

6

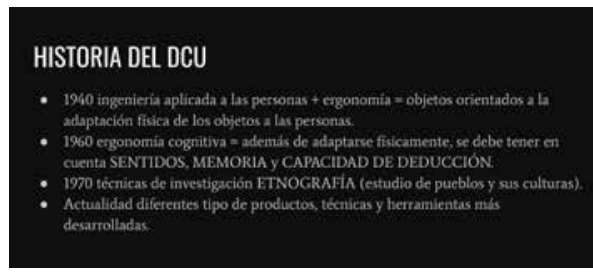

5

Figura 4. Aula virtual. Recursos del docente: Conceptos de Diseño y Definición y características. Recursos adicionales: Bibliografía general y artículos específicos del DCU. (Autor, 2021)

Figura 5. Recursos del docente: Concepto, objetivo e historia del DCU. (Autor, 2020) Figura 6. Resultado, infografía: DCU definición, características y etapas; estudiante Ariana Sosa. (Autor, 2020) 
La sintética se la propone en la parte práctica del proyecto desde las fases de investigación, desarrollo creativo y comprobación; después de la explicación de los métodos de diseño en las distintas fases por parte del docente, los estudiantes los ejecutan, es decir las aplican, así crean y acrecientan el conocimiento: sobre el tema del proyecto y las diferentes herramientas y métodos de diseño. Mostramos a continuación los recursos y los resultados de su aplicación en las distintas fases.

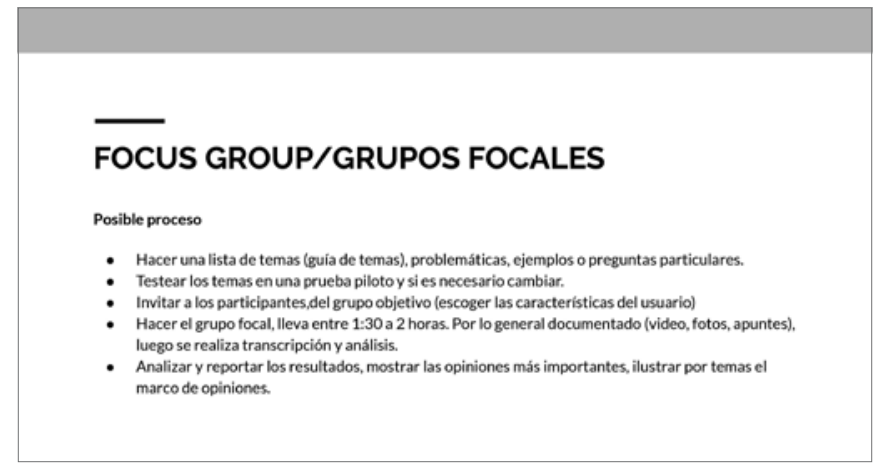

7A

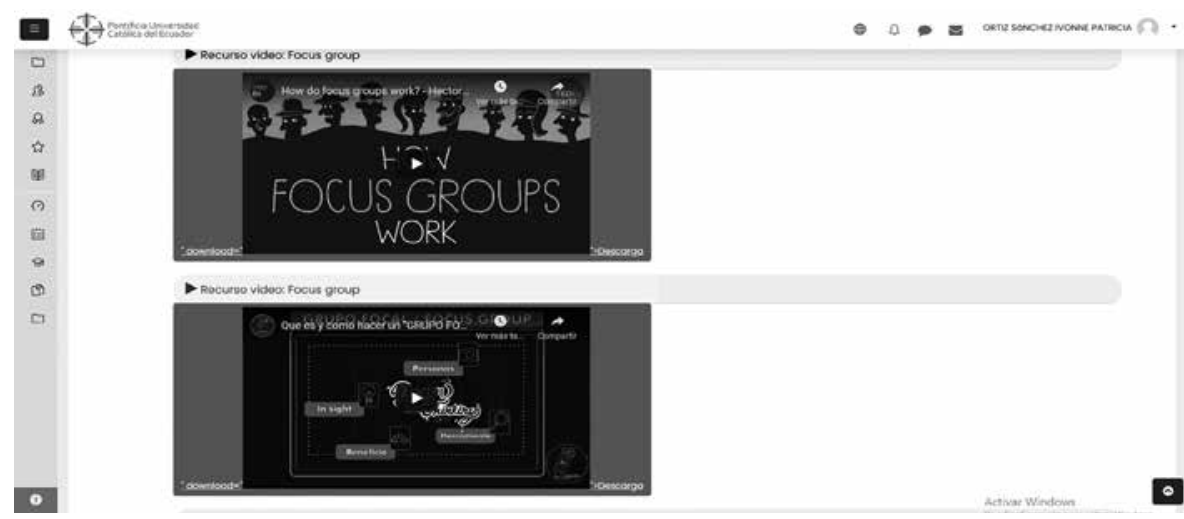

$7 \mathbf{B}$

Figuras 7A y B. Recursos del docente, fase de investigación: Métodos entrevista y focus group, presentación y videos de soporte en el aula virtual. (Autor, 2020) 


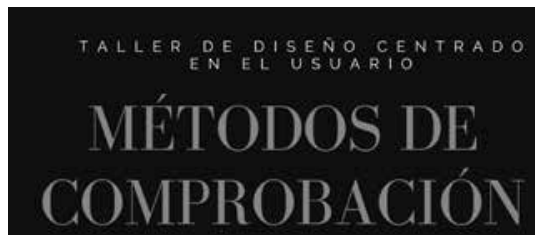

\section{Evaluación de usabilidad}

Para esta evaluacion se presento a los usuarios el producto para tener un feedback real acerca de como percibian al objeto en cuanto a su usabilidad, esto nos permite poder evaluar la experioncia de uso que tienen las personas y sili .
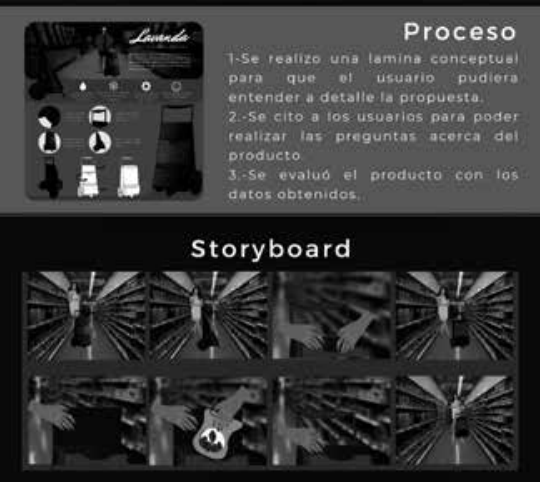

\section{Aplicación}

Se hizo las siguientes preguntas a los usuarios mediante una reunion de $z 00 \mathrm{~m}$ despues de presentar it propuest: - ¿Que piensas del producto - tentendisto a primera vista como funcionar - LQue no te gusto?

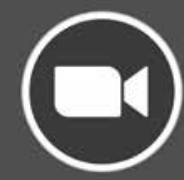

Resultados

Si se entendio la ¿Qué elementos ¿Que se evaluo? usabilidad Qué elementos toman como : vobisad
señales de uso? tosvasumos entandanch in

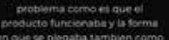

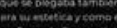

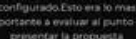

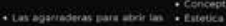
- Sospeguns a los usces. . manos

10
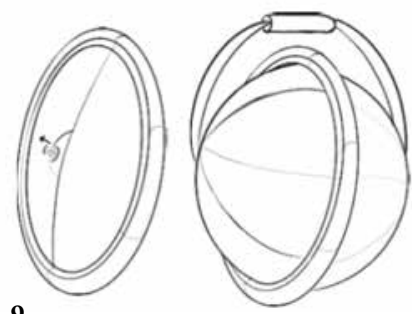

Metáfora

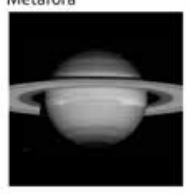

Analogía
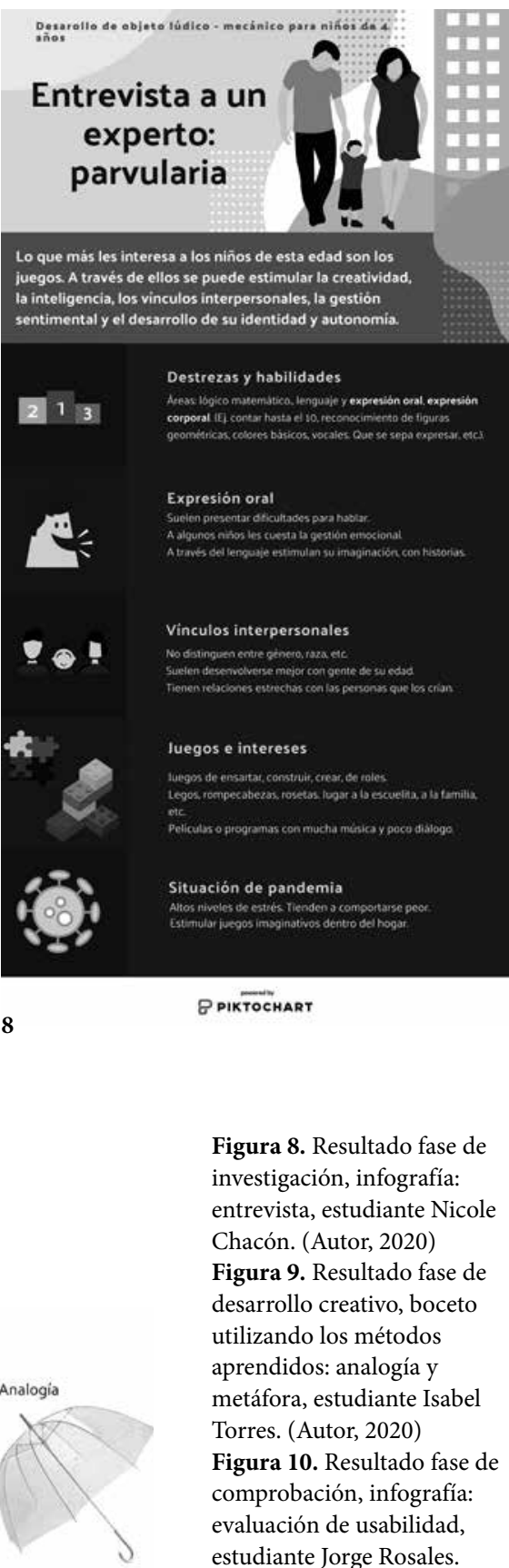

8

P PIKTOCHART

Figura 8. Resultado fase de investigación, infografía: entrevista, estudiante Nicole Chacón. (Autor, 2020)

Figura 9. Resultado fase de desarrollo creativo, boceto utilizando los métodos aprendidos: analogía y metáfora, estudiante Isabel Torres. (Autor, 2020)

Figura 10. Resultado fase de comprobación, infografía: evaluación de usabilidad, estudiante Jorge Rosales. (Autor, 2020) 


\section{Esquema del aula}

\section{Clase de conceptos teóricos}

Cuando se desarrollan los conceptos teóricos se utiliza la estrategia del aula invertida ${ }^{1}$. Se facilita recursos bibliográficos, videos y referencias para que los estudiantes lo trabajen autónomamente; dentro de la clase se genera una mesa redonda para debatir sobre los temas estudiados, generar criterios, reforzar conocimientos y solventar dudas. Con los conceptos claros se procede a generar infografías, mapas mentales o fichas que permiten revisar los temas tratados cuando es necesario dentro del nivel o en los futuros semestres.

\section{Clase del proceso proyectual}

Durante el desarrollo del proyecto la estrategia cambia, primero la docente explica los conceptos, proporciona ejemplos para ilustrar los procesos; los estudiantes asimilan la información y la prueban, una vez aplicado el conocimiento se reflexiona sobre los resultados. En conjunto con él estudiante se genera un análisis crítico para analizar la organización del proceso, los objetivos planteados, la aplicación y los resultados obtenidos.

Se busca fomentar la experimentación para alcanzar aprendizaje significativo, además la reflexión y la crítica como habilidades y destrezas del diseñador en el ejercicio profesional.

\section{Estrategias en el aula}

Se han generado varias estrategias para trabajo en el aula entre las principales están: (1) Motivar a los estudiantes a probar nuevas herramientas, aventurarse a experimentar, (2) reconocer los errores y el aprendizaje que dejan, (3) analizar y criticar sus propias propuestas de manera objetiva con guía de la docente, (4) reconocer el proceso, no solo centrarse en el resultado del proyecto.

Sobre el trabajo de los estudiantes (5) fomentar el trabajo colaborativo, donde la generación de conocimiento es responsabilidad de todos los involucrados, (6) generar información en conjunto y obtener información para cada proyecto individual.

\section{La virtualización de los talleres}

De los cuatro periodos que lleva implementado el TDCU solo uno fue dado en presencialidad, los demás se han desarrollado de manera virtual, esto llevo a un rápido análisis de la asignatura, para la implementación de estrategias que permitan alcanzar los resultados de aprendizaje en esta nueva modalidad.

La virtualidad facilita las clases teóricas, es decir la primera parte de la asignatura sobre los conceptos básicos, ya que a través de recursos, presentaciones, videos, lecturas y ejercicios 
los estudiantes perciben mejor el contenido y lo asimilan, además que las herramientas en línea como picktochart, creately, adobe spark, canva, entre otros facilitan los ejercicios para los estudiantes, obteniendo óptimos resultados. Se propone ejercicios que permita a los estudiantes sintetizar la información y se generan recursos como infografías, fichas y mapas mentales que pueden revisar constantemente durante el semestre.

Durante las fases prácticas, de desarrollo del proyecto la dinámica se vuelve mas compleja ya que los métodos requieren de cierto análisis y modificaciones para hacerlo de modo virtual.

Se logró su aplicación remota identificando en el círculo de amigos y familiares los usuarios pertinentes por proyecto. Se ha recurrido a las herramientas digitales de video conferencia zoom y teams para hacer los encuentros y aplicación de métodos de investigación y comprobación.

\section{MAPAS CONTEXTO
RELEVANTE PARA EL
DISEÑO}

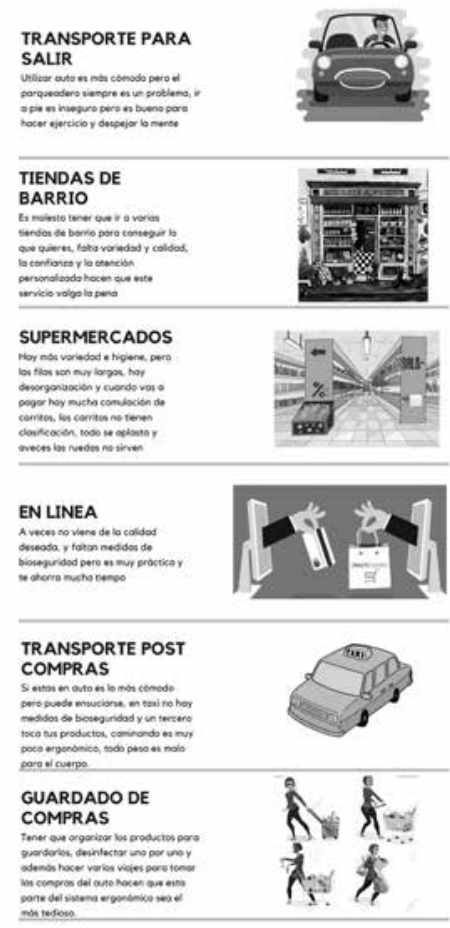

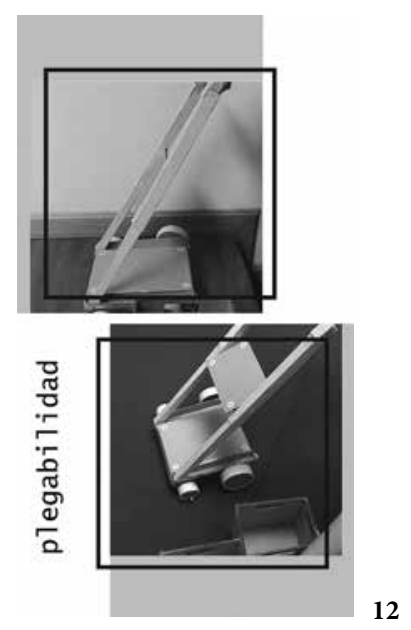

Figura 11. Resultado fase de investigación, infografía: mapa de contexto, método aplicado a través de la experimentación y diálogo con sus familiares. Estudiante Nicole Chacón. (Autor, 2020)

Figura 12. Modelos de estudio Estudiante Nicole Chacón.

(Autor, 2020) 
En cuanto a los métodos creativos herramientas como jamboard, stormboard, entre otras permiten el trabajo colaborativo, para ejercicios que motiven la creatividad, y el desarrollo de ideas y propuestas. Se pueden utilizar herramientas manuales o digitales que permitan la comunicación de las ideas de los estudiantes. Además, se propone métodos individuales y colectivos que enriquecen la generación de ideas.

Sobre el desarrollo de modelos de estudio y prototipos rápidos, existen limitantes en el acceso de materiales, dependiendo de la ubicación de los estudiantes, esto permite ser recursivo e identificar lo que tengo en casa y como los puedo aprovechar, analizando las características del material y los requerimientos del objeto.

Una vez desarrollada la propuesta a detalle, cerca más al objeto definitivo; se requieren comprobaciones que pueden ser realizadas de manera virtual o presencial dependiendo del requerimiento, por ejemplo, una comprobación como el método y herramienta PREMO que mide las reacciones y emociones de la persona respecto al objeto se puede realizar de manera virtual; se muestran imágenes del objeto, se explica brevemente y las personas lo validan dando su respuesta.

Con relación a validaciones como la de usabilidad y la interacción es imposible realizarla de manera virtual, con esta debemos reconocer en el entorno cercano quien está dentro del grupo objetivo para que interactúe con el objeto y lo valide.

Cada método aplicado requiere de planificación, ejecución, recopilación, análisis y resultados para lo que se realizan registros exhaustivos, donde se obtiene la información requerida.

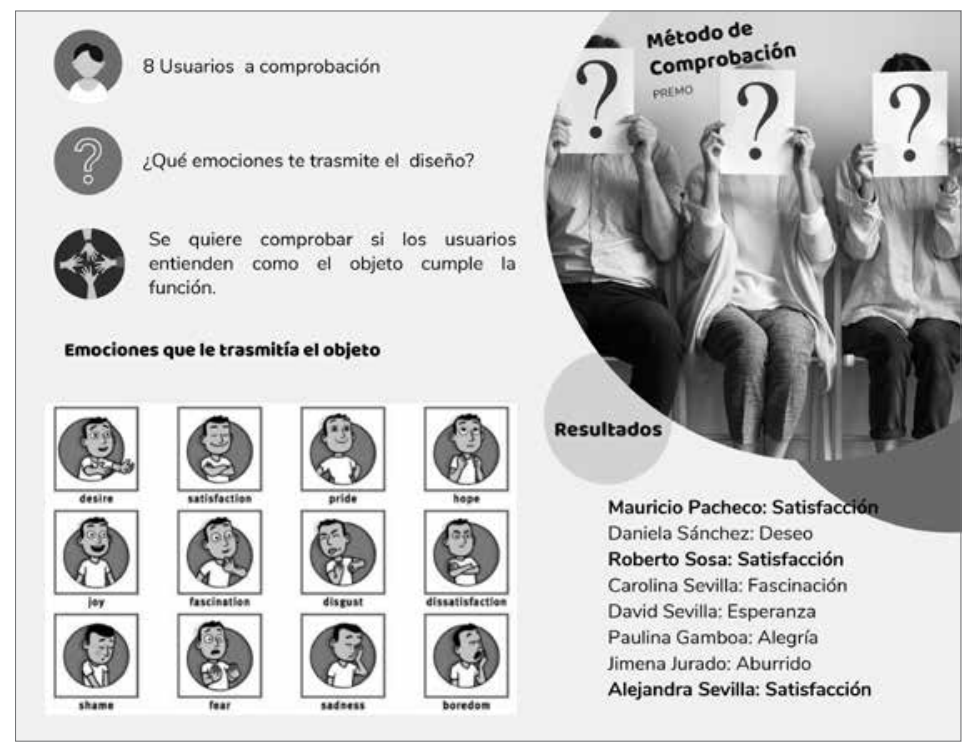

Figura 13. Resultado fase de comprobación, infografía: PREMO, método aplicado virtualmente. Estudiante Ana María Sevilla. (Autor, 2020) 


\section{Aciertos}

Los aciertos de este proceso se han dado por la fuerte base teórica, pedagógica y metodológica que se ha construido en la asignatura. Entre los que podemos mencionar: (1) observar nuestro entorno, la cotidianidad que nos rodea objetualmente y en cuanto a actividades las pasamos por alto, estar en casa nos permite identificar los problemas y recursos que tenemos a nuestro alcance; (2) análisis y propuesta de aplicación de métodos de todas las fase de manera virtual y presencial según la pertinencia de cada uno; (3) Se han desarrollado diversidad de temas en los proyectos: Vajilla para comida típica de la provincia de Manabí, productos para mejorar el teletrabajo, Transporte para compras en Pandemia y productos para la agricultura urbana; estos temas se han analizado tomando en cuenta que los estudiantes tengan acceso a la investigación y la experimentación. (4) Promover la recursividad de los estudiantes con el uso de materiales que existen en casa o de fácil acceso para la generación de los modelos y prototipos de experimentación.

\section{Limitaciones y errores}

En la virtualización de la asignatura se han presentado limitaciones y errores entre los cuales están: (1) limitación con los estudiantes ya que cada uno percibe la información de diferente manera, para alcanzar un resultado óptimo en la mayoría de los estudiantes ha tomado dos periodos, mediante análisis de resultados y ajuste de estrategias. (2) No se ha alcanzado una homogenización en la complejidad de los proyectos desarrollados, existe disparidad entre los proyectos de los estudiantes; (3) el proceso pedagógico depende muchísimo de la disposición de los estudiantes, no se alcanzan todos los objetivos con todos los estudiantes; (4) la metodología aplicada requiere la capacidad de los estudiantes a aventurarse a aplicar los instrumentos, el aprendizaje depende de ellos.

\section{Conclusiones}

A modo de conclusión las propuestas teórica, pedagógica y metodológica son constantemente revisadas lo que ha permitido una construcción sólida y continua. Se busca ampliar las referencias bibliográficas y los recursos generados para que los estudiantes tengan un amplio espectro de información para desarrollar sus criterios.

Se ha construido una propuesta de carrera basada en los diferentes énfasis que ha desarrollado el Diseño de Productos, esto permite reconocer un enfoque, y abordar los diferentes procesos metodológicos del proyecto, así como la aplicación de métodos, técnicas y herramientas dentro de las distintas fases del proyecto.

El Taller de Diseño Centrado en el Usuario posee una estructura coherente para el proceso de aprendizaje de los estudiantes, además de evidenciar claros resultados de aprendizaje. Las estrategias de aprendizaje se centran en el protagonismo del estudiante dentro del pro- 
ceso para proveerlo de habilidades, capacidades y destrezas que le aporten a su formación profesional.

Para la virtualización del TDCU se ha pensado en estrategias de enseñanza y para la aplicación de los instrumentos de manera virtual; se ha fomentado la recursividad de los estudiantes y se han propuestos proyectos relevantes a las problemáticas actuales.

Los ejemplos mostrados pertenecen a la construcción realizada por la docente y a los resultados alcanzados por los estudiantes de la promoción del segundo semestre 2020, los cuales muestran los procesos y, la capacidad de comunicación de cada estudiante.

Los aciertos alcanzados superan los errores cometidos, que nos afirma el éxito de la virtualización del taller, los proyectos desarrollados en el TDCU se han analizado en base a la accesibilidad de información y capacidad para comprobar el proceso de diseño. Se analizará y reflexionará sobre las limitaciones y errores para mejorar el proceso educativo. Al finalizar el presente semestre, con el cual se cumplen los cuatro periodos desde su implementación, se analizará en conjunto con el comité de carrera los resultados obtenidos para determinar las buenas prácticas, los ajustes pertinentes y su futura proyección.

\section{Notas}

${ }^{1}$ Aula invertida es un modelo pedagógico que transforma ciertas partes del proceso del aprendizaje llevándolos fuera del aula mientras incrementa o da mayor importancia a otras actividades dentro del aula. De esta manera los alumnos trabajan por un lado en un espacio individual y a su ritmo fuera del aula, mientras que el aprendizaje dentro del aula se vuelve mucho más dinámico e interactivo, donde los educadores guían y conducen a sus alumnos en dicho aprendizaje a través de la colaboración. (Capacitación en ambientes virtuales, 2020)

\section{Bibliografía}

Antunes, P.; Cunca, R. (2019). Product Innovation: A Collaboration between Sciences and the Arts. Convergencias, revista de investigación y enseñanza de las artes.

BECERRA, Ovídio Rincon (2016). Ergonomía y procesos de diseño: Consideraciones metodológicas para el desarrollo de sistemas y productos. Bogotá: Pontificia Universidad Javeriana.

Brandes, U., Stich, S.; Wender, M. (2013). Design by Use: The Everyday Metamorphosis of Things. Basel: De Gruyter.

Claxton, G. (2001). Aprender: El reto del aprendizaje continuo. Barcelona: Paidós.

Duran, JC. (2004). Teorías del aprendizaje y modelos pedagógicos. Quito: Universidad Central del Ecuador

Grand, S., \& Jonas, W. (2012). Mapping design research. Basel: Birkhäuser.

IDEO (2009). Diseño centrado en las personas. 
IDEO (Firm). (2003). IDEO method cards. Place of publication not identified: IDEO.

Johnson, J. (2014). Designing with the mind in mind: Simple guide to understanding user interface design guidelines.

Johnson, J. (2010). Designing with mind in mind: Simple guide to understanding user interface design rules. Amsterdam [u.a.: Morgan Kaufmann/Elsevier.

Koskinen, I. K. (2012). Design research through practice: From the lab, field, and showroom. Waltham, MA: Morgan Kaufmann/Elsevier.

Kuniavsky, M., \& Moed, A. (2012). Observing the User Experience: A Practitioner's Guide to User Research. San Diego, CA, USA: Elsevier Science \& Technology Books.

Lupton, E., Carpentier, T., \& Lambert, T. (2014). Beautiful users: Designing for people. New York: Princeton Architectural Press - Cooper Hewitt, Smithsonian Design Museum.

Lidwell, W., Holden, K., Butler, J., \& Safari, an O'Reilly Media Company. (2010). Universal Principles of Design, Revised and Updated. Maeda, J. (2006). The laws of simplicity. Cambridge, Mass: MIT Press.

Martin, B., \& Hanington, B. M. (2018). The pocket universal methods of design: 100 ways to research complex problems, develop innovative ideas and design effective solutions.

Marín, T. J. M., \& Torrent, R. (2016). Breviario de diseño industrial: Función, estética y gusto. Madrid: Cátedra.

Moggridge, B. (2007). Designing interactions. Cambridge, Mass: MIT Press.

Norman, D. A. (2016). Living with complexity.

Norman, D. A. (2007). Emotional design: Why we love (or hate) everyday things. New York: Basic Books.

Pratt, A., \& Nunes, J. (2013). Diseño interactivo: Teoría y aplicación del DCU. Barcelona: Oceano.

PUCE (2018). Documento del Rediseño de la Carrera de Diseño de Productos. Quito. PUCE

PUCE (2019). Documento del Ajuste del Rediseño de la Carrera de Diseño de Productos. Quito. PUCE

PUCE (2021). Sílabo del Taller de Diseño Centrado en el Usuario. Quito. PUCE

Rodríguez, M. L. (2010). Diseño: Estrategia y táctica. México: Siglo XXI

Rubin, J., \& Chisnell, D. (2008). Handbook of usability testing: How to plan, design, and conduct effective tests. - Includes index. Indianapolis, Ind: Wiley Pub.

Technische Hogeschool Delft. (2017). Delft design guide.

Wender, M., Brandes, U., \& Stich, S. (2013). Design by Use: The Everyday Metamorphosis of Things. (Design byuse.) Basel: De Gruyter.

Abstract: This paper aims to present the User-Centered Design workshop (TDCU),
analyze his theoretical, pedagogical, and methodological proposals developed during
the teaching experience on course and a the develop of the User centered project. The
pedagogical and methodological strategies applied on the design teaching will be
analyzed in detail focusing on obtained results, successes and errors during the four
academic periods, on face to face and virtual lessons, since this workshop was stablished. 
It is articulated on a review of the Product Design career history, the construction of the pensum redesign that generated the workshop and a explanation of the structure of the course. The pedagogical principles based on the cognitivism that are being applied will be developed and the methodological proposal stated on this learning and teaching processes will be explained. Finally, once the framework is presented, it will discuss the applied strategies to the virtualization of the class: faced challenges, positive points and mistakes in order to analyze and conclude the future of the course.

Key words: Workshop of User Centered Design - methodology - learning outcomes pedagogy.

Resumo: O presente artigo incidirá na temática do Workshop de Design Centrado no Utilizador (TDCU), para analisar as propostas teóricas, pedagógicas e metodológicas que se vão construindo no âmbito da disciplina, bem como no projecto de Design com ênfase no utilizador. As estratégias metodológicas e pedagógicas aplicadas ao ensino de Design e os resultados obtidos neste processo de construção do conhecimento, bem como os acertos e erros que se geraram durante a implementação desta disciplina, nos quatro períodos em que foi ministrada a cara a cara e virtual. É feita uma recontagem sobre a Carreira de Design de Produto e a construção do Redesign de que nasce a proposta da TDCU, daí se conhece a estrutura do tema; São desenvolvidos os princípios pedagógicos baseados no cognitivismo que são utilizados nesta cadeira, bem como a proposta metodológica que é aplicada neste processo de ensino e aquisição de conhecimentos. Uma vez desenvolvido este framework, trata-se das estratégias adotadas para a virtualização do assunto, os desafios enfrentados, os sucessos alcançados, as limitações e erros cometidos, de forma a analisar e concluir o futuro do assunto.

Palavras-chave: Workshop de Design Centrado no Usuário (TDCU) - Metodologia, Resultados de aprendizagem - Pedagogia.

[Las traducciones de los abstracts fueron supervisadas por el autor de cada artículo] 